\title{
ReMed: Jahresbericht 2018
}

ReMed, Leitungsausschuss

\section{Kennt ihr ReMed - das Unterstützungs- netzwerk für Ärztinnen und Ärzte?}

ReMed ist ein Unterstützungsnetzwerk für Ärztinnen und Ärzte, welches im Jahr 2010 schweizweit eingeführt wurde. Es verbreitet Wissen und Erfahrungen über Gesundheitsförderung und Prävention bei Ärztinnen und Ärzten und sensibilisiert diese für die eigene Gesundheit. In Krisensituationen unterstützt ReMed Ärztinnen und Ärzte mit einem breiten Angebot. Ziele sind die Erhaltung der Gesundheit und ärztlichen Funktionalität sowie die Gewährleistung der Patientensicherheit und der hohen Qualität in der medizinischen Versorgung.

\section{Erstberatung und Vernetzung - das Hauptangebot}

Die Gesundheit der Ärztin, des Arztes steht bei ReMed im Zentrum, mit besonderer Beachtung berufsspezifischer Risiken und der Prävention. Die Stellung der ratsuchenden Ärztin, des ratsuchenden Arztes entspricht, wie oben beschrieben, der Stellung eines Patienten auch wenn damit nicht gesagt wird, dass die betreffende Ärztin resp. der betreffende Arzt an einer Krankheit leidet.
ReMed sucht während des ganzen Prozesses eine Vernetzung zu bestehenden Angeboten und die Zusammenarbeit mit allen Fachleuten, welche die Zielsetzungen von ReMed teilen. Mit dem Ziel einer sorgfältigen (Erst-) Beratung nimmt ReMed mit der ratsuchenden Kollegin, dem ratsuchenden Kollegen innert 72 Stunden Kontakt auf und versucht, die Situation zu verstehen und erarbeitet gemeinsam Lösungen.

Der überwiegende Anteil der ReMed Nachfragen führt zu Vorschlägen und wenn nötig zur Vermittlung von Begleitung, Unterstützung oder Therapien durch Hausärzte, Spezialärzte (z.B. Psychiater-Psychotherapeuten) sowie durch weitere Fachkräfte (z.B. Advokaten, Finanzberater).

\section{Kontaktaufnahmen im vergangenen Jahr}

Gegenüber dem Vorjahr haben die Kontaktaufnahmen leicht zugenommen und sind auf 143 angestiegen. Am meisten Kontaktaufnahmen erfolgten im Monat November. Diese stetige Zunahme beweist die Notwendigkeit der ReMed-Dienste deutlich. Bei den Kontaktaufnahmen, welche von einem ärztlichen Mitglied des Leitungsausschusses beantwortet wurden, erfolgten 114 Anfragen aus der Deutschschweiz, 28 Fälle aus der

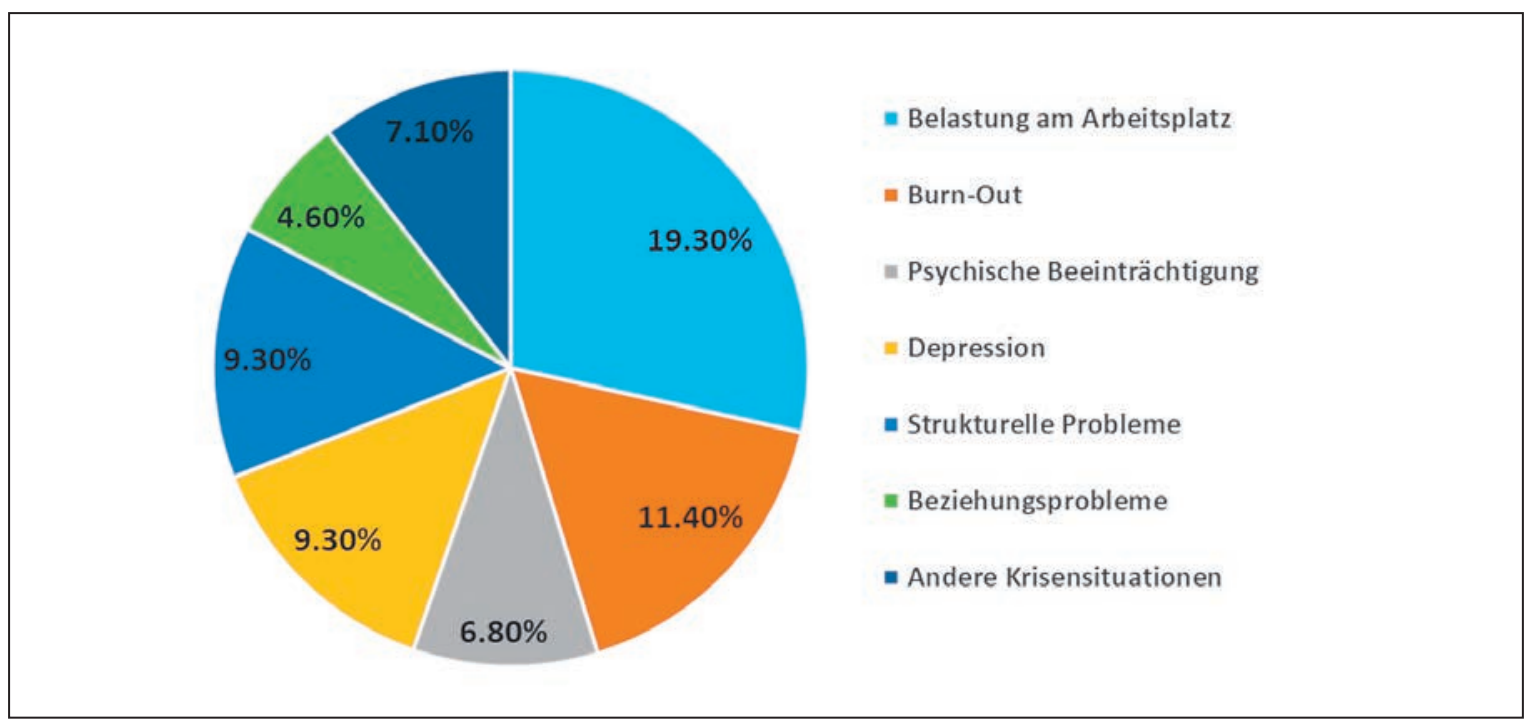

Abbildung 1: Gründe für Kontaktaufnahme bei ReMed (Mehrfachnennungen möglich). 
Romandie und 1 Fall aus dem Tessin. Die Kontaktsuchenden waren zu $70 \%$ weiblich und 30\% männlich. Die Mehrheit der Kontaktsuchenden kam aus dem stationären Bereich. Die Problematik «Belastung am Arbeitsplatz» gefolgt von den Problemen «Burn-Out» und «Depression» waren die Hauptgründe der Kontaktaufnahme (vgl. Abb. 1).

\section{ReMed Programm - Vernetzung}

Die Belastung am Arbeitsplatz, Burnout und die Doppelbelastung von Mutterschaft und Beruf sind die häufigsten genannten Gründe für die Kontaktaufnahme bei ReMed. Eine Zunahme der Problematik Arbeit/Familie (Doppelbelastung), Gleichberechtigung und Karriere/Teilzeitarbeit hat auch der Verband Schweizerischer Assistenz- und Oberärztinnen und -ärzte (VSAO) beobachtet. ReMed und der VSAO vereinbarten im 2018, gemeinsam solche Schnittstellen zu beobachten, $\mathrm{zu}$ pflegen und Lösungsangebote zu erarbeiten. Das Netzwerk von ReMed wurde im 2018 wiederum mit Erstberatenden erweitert und verstärkt. Es bestehen wichtige Kontakte zu Organisationen in der Deutschschweiz wie auch in der Romandie. Man ist bestrebt, die Angebote laufend zu optimieren und zu erweitern, so werden nächstens auch Gesprächsrunden in Spitälern der Romandie angeboten.

\section{ReMed - Coaching-Gruppen neu auch in St. Gallen}

Ende 2018 startete erstmals auch eine CoachingGruppe in St. Gallen. In Bern und in Zürich finden seit 2016 solche Gesprächsrunden statt. Unter fachlicher, kompetenter Anleitung wird Unterstützung für den herausfordernden Berufsalltag geboten. Arbeitsbezogene Konflikte und Schwierigkeiten werden the- matisiert und entsprechende Lösungen gemeinsam diskutiert und erarbeitet. Informationen zu den Gruppenangeboten finden Sie unter www.swiss-remed.ch.

\section{ReMed - Gegenwärtig sein}

Das Unterstützungsnetzwerk repräsentierte mit Workshops und Darbietungen sein Angebot an zahlreichen Kongressen und Lehrveranstaltungen sowie in Institutionen und konnte das Programm einem immer grösser werdenden Kreis differenziert bekannt machen. Diese Auftritte leisteten damit weiter grossen Beitrag zur Enttabuisierung der beruflichen ärztlichen Belastungen. Regelmässig sind im vergangenen Jahr wieder eindrückliche Testimonials (Erfahrungsberichte) in der Schweizerischen Ärztezeitung erschienen. Das Aufzeigen, wie ReMed Betroffenen helfen konnte, erfährt bei den Lesern grossen Anklang, wie es die positiven Rückmeldungen jeweils deutlich machen. Auch in den Medien war ReMed 2018 präsent und konnte entsprechende Artikel veröffentlichen.

\section{ReMed - Erfahrungsberichte und Dokumentationen}

Besuchen Sie die Homepage www.swiss-remed.ch und finden Sie interessante Erfahrungsberichte und Dokumentationen sowie alle wertvollen Informationen über das Unterstützungsnetzwerk.

Das nationale Programm ReMed mit dem Leitungsausschuss und den Netzwerkmitgliedern wird auch im Jahr 2019 für Ärztinnen und Ärzte in Krisensituationen da sein. Die betroffenen Ärztinnen und Ärzte können über die 24-Stunden-Hotline (0800 0 73633) oder per help[at]swiss-remed.ch Beratung und Hilfe anfordern. 